\title{
Foreperiod and range effects on time interval categorization
}

\author{
Vincent Laflamme ${ }^{1}$ - Dan Zakay ${ }^{2}$. \\ Pierre-Luc Gamache ${ }^{1} \cdot$ Simon Grondin ${ }^{1}$
}

Published online: 29 May 2015

(C) The Psychonomic Society, Inc. 2015

\begin{abstract}
One factor influencing the perceived duration of a brief interval is the length of the period preceding it, namely the foreperiod (FP). When multiple FPs are varied randomly within a testing session, longer FPs result in longer perceived duration. The purpose of this study was to identify what characteristics modulate this effect. In a task where participants were asked to categorize the duration of target intervals with respect to a $100-\mathrm{ms}$ standard, the FPs were distributed over a $150-$, 300-, or 900-ms range with the midpoint (1000 ms) of these distributions being kept constant. The results indicate that the effect of the length of variable FPs on perceived duration was much stronger in the $900-\mathrm{ms}$ range condition. More specifically, this effect is due to the differences between the shortest FPs. The results also reveal that, overall, there are more short responses in the 300-ms condition than in the other range conditions. Moreover, the data reveal that the narrower the distribution, the better the discrimination. One interpretation of the main result (range effect) is that a wider distribution leads to an increased prior uncertainty towards the foreperiod length.
\end{abstract}

Keywords Perception and action · Attention and memory · Categorization

One of the oldest and most robust findings in the field of cognitive psychology is the fact that the length of the

Vincent Laflamme

vincent.laflamme.1@ulaval.ca

Simon Grondin

simon.grondin@psy.ulaval.ca

1 École de psychologie, Université Laval, 2325 rue des Bibliothèques, Québec, QC, Canada G1V 0A6

2 Inter-Disciplinary Center, Herzeliya, Herzeliya, Israel foreperiod (FP) - the temporal interval that precedes the presentation of an imperative stimulus - can influence severely one's reaction time (RT) to that stimulus. In particular, it has been found that, when FP lengths vary randomly from trial to trial, RT will be longer when the target stimulus is preceded by the shorter FP than when it is preceded by the longer FP (Drazin, 1961; Karlin, 1959; Klemmer, 1956; Los \& Van den Heuvel, 2001; Niemi, 1979; Niemi \& Näätänen, 1981). On the other hand, when FPs remain constant within a block of trials, RTs increase with FP length (Klemmer, 1956; Niemi \& Nätänen, 1981).

One of the most accepted explanations for the FP effect on RT pertain to temporal preparation and temporal uncertainty. Succinctly, more preparation to respond to the imperative stimuli leads to shorter RTs. The level of preparation one is able to muster before the imperative stimulus depends on the length of the FP. When FPs vary randomly from trial to trial, participants are less prepared to respond to the imperative stimulus when it follows shorter FPs than when it follows longer FPs. Indeed, as FPs elapse, the conditional probability that the imperative stimulus will occur shortly increases. Conversely, when FPs are kept constant within block, preparation to respond to the imperative stimulus is better for shorter FPs than longer FPs. The main reason for that is that temporal uncertainty about the FP length increases with the length of constant FPs above a certain optimal value (Klemmer, 1956).

Recent studies suggest that the FP effect phenomenon may not be exclusive to simple RT paradigms. Indeed, Mo and George (1977) discovered that the FP length influences the perceived duration of a following time interval. Using a variable FP paradigm, they found that target intervals following shorter FPs were consistently underestimated (Experiments 1, 2, and 3) and under-reproduced (Experiments 4 and 5) compared with target intervals following longer FPs. According to these authors, such results show that the FP length affects 
perceived duration by influencing the preparation to detect the onset of the target interval. As with simple RT experiments following the same variable FP paradigm, the participants' temporal preparation increases as FP elapses. Recent successful replications of the variable FP effect on time perception have attested the robustness of this empirical effect as well as the significance of the temporal preparation explanation (Bendixen, Grimm, \& Schröger, 2005; Gamache, Grondin, \& Zakay, 2011; Grondin \& Rammsayer, 2003; Los \& Horoufchin, 2011). This type of manipulation also has been used for studying time-order judgments (Bausenhart, Rolke, \& Ulrich, 2008).

It would be tempting to conclude that both phenomenathe FP effect on simple RT and the FP effect on perceived duration - are caused by alterations of common core mechanisms. However, although the topic of the FP effect on time perception has been explored, it has been subjected so far to considerably less empirical inquiry than its simple RT equivalent. In order to determine whether both phenomena are caused by a common mechanism - namely, temporal preparation - it is important to verify that the FP effect on perceived duration exhibits the same fundamental properties as the FP effect on RT. One example of such relevant property is the well-known fact that the random FP effect on RT is modulated by the within-block variability of the FPs: the difference of RT
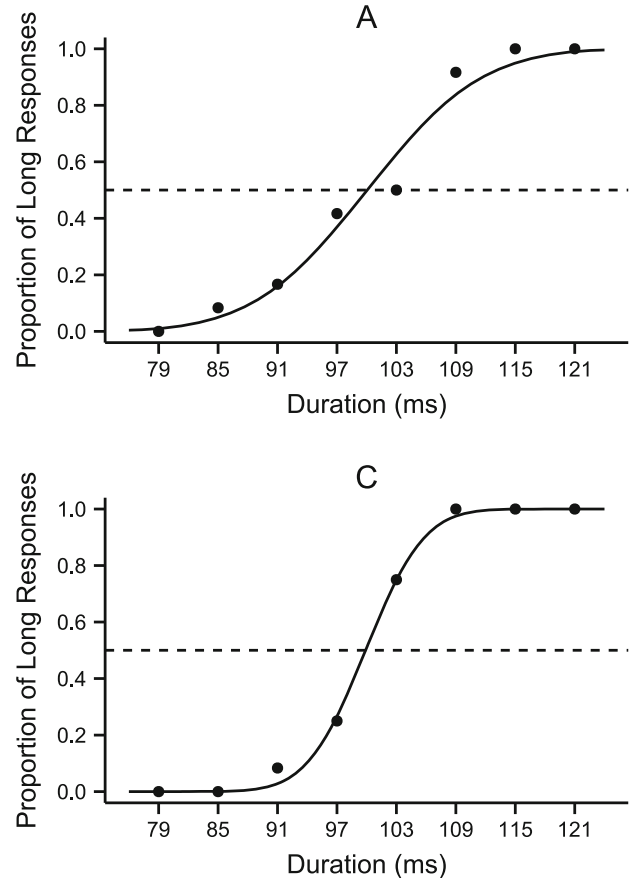

Fig. 1 Four examples of psychometric function fittings. Each panel plots the proportion of long responses as a function of target duration and shows the resulting fitted cumulative Gaussian curve. The bisection point (BP) is defined as the target duration value where horizontal line intersects with the fitted curve. Thus, the BP for the data in panel $\mathbf{A}$ $(100 \mathrm{~ms})$ is lower than that for the data in panel $\mathbf{B}(112 \mathrm{~ms})$. However, it can be seen that the target durations in panel $\mathbf{A}$ were generally judged as between the shortest and longest FP conditions increases with the variance of the FPs (Drazin, 1961; Elliott, 1973; Klemmer, 1956).

The main objective of the present study was to verify whether this particular property of the FP effect on RT is applicable to the temporal perception paradigm. We used the same general experimental design as Grondin and Rammsayer (2003) and Gamache et al. (2011), i.e., a task in which participants are asked to judge whether a given temporal interval delimited by two tones is shorter or longer than a $100-\mathrm{ms}$ standard interval. It should be noted that, in this kind of experimental design, the participants' subjective duration of the target intervals is measured by the bisection point (BP). It is defined as the target duration that gives rise to "longer" responses exactly $50 \%$ of the time (Fig. 1A and B).

\section{Temporal preparation and the internal clock model of time perception}

The idea that FP length could influence the perceived duration through the process of temporal preparation is consistent with the prevailing literature on time perception. Indeed, it is wellknown that attentional processes play an important role in time perception (Brown, 1997; Casini, Macar, \& Grondin,
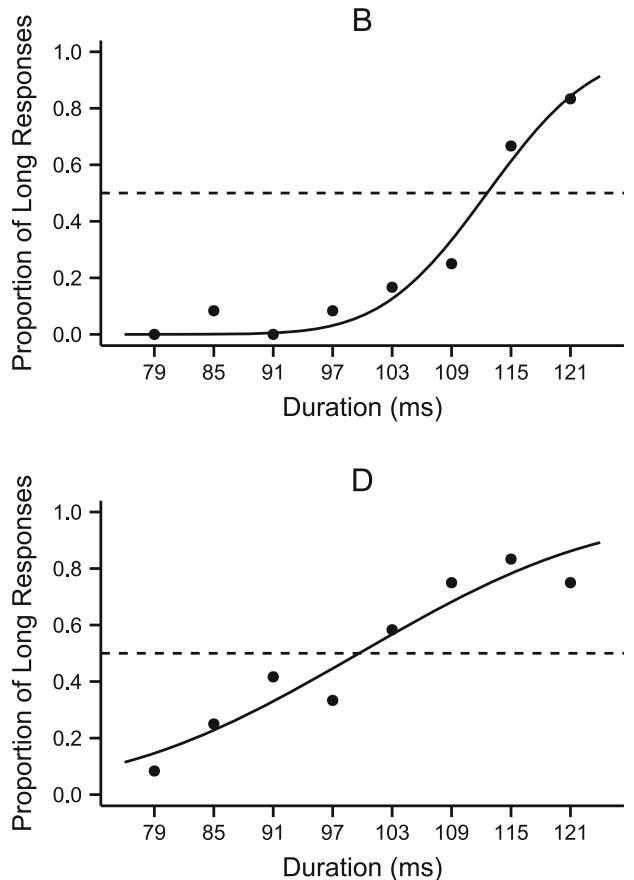

"long" more often than those in panel B. This highlights the negative relationship between $\mathrm{BP}$ and perceived duration. Whereas the BPs for the data in panels $\mathbf{C}$ and $\mathbf{D}$ are equal $(100 \mathrm{~ms})$, the proportion of "long" responses are much closer to chance level $(50 \%)$ for the data in panel D than for the data in panel C. This feature of the data is captured by the estimated standard deviation parameter of the fitted Gaussian curve (4.5 $\mathrm{ms}$ in panel $\mathbf{C}$ and $20 \mathrm{~ms}$ in panel D) 
1992; Grondin \& Macar, 1992; Macar, Grondin, \& Casini, 1994). The most widely cited model of time perception, the information processing version of the Scalar Expectancy Theory (SET - Gibbon, Church \& Meck, 1984), as well as its extension, the Attentional Gate Model (Block \& Zakay, 1996, 1997; Zakay \& Block, 1996, 1997), acknowledge specifically the importance of attentional processes. According to SET, perceived duration results from the accumulation of pulses by a pacemaker module. In order for pulses to be counted, an attentional switch, whose latency is determined by one's attention to time, must be closed. Thus, greater latency before the beginning of the timekeeping activity results in fewer accumulated pulses and, consequently, shorter perceived duration of the target interval. If, as Mo and George (1977) surmised, better temporal preparation leads to faster detection of the onset of the target interval, it means that the process of pulses' accumulation begins earlier and duration is perceived as longer.

\section{Prior and posterior temporal information}

When discussing the effect of temporal preparation on RT or time perception, it is useful to distinguish two types of temporal information: prior and posterior temporal information (Mo, 1990). In this context, prior information refers to the knowledge one extracts about the random distribution of the FPs. As participants complete trials in a variable FP block, they learn (implicitly or explicitly) that they will only be subjected to four different FPs. Posterior information, on the other hand, refers to the knowledge concerning the current FP that the observer can deduce while the FP is elapsing; it is essentially the subjective conditional probability that the onset marker of the target interval will occur given the time elapsed since the beginning of the current trial (Mo, 1990). For example, if participants know that the FP can last 600, 900, 1200, or $1500 \mathrm{~ms}$ and that the time elapsed since the beginning of the trial is longer than $1200 \mathrm{~ms}$, they can conclude with certainty that the target interval will begin within the next $300 \mathrm{~ms}$. Note that posterior information is built upon prior information. Another important property of prior and posterior temporal information is that they are complementary (Mo, 1990). When posterior information about the FP is low, such as at the very beginning of the trial, the participants' level of temporal preparation will solely depend on what they learned about the FP distribution from past trials. However, as posterior temporal information about the FP increases (i.e., as the FP elapses), it becomes more and more central to the participants' level of preparation.

Looking at the FP effect on time perception from the perspective of temporal preparation, it could be said that the core findings in the literature regarding the variable FP effect mostly pertain to the effect of posterior temporal uncertainty on perceived duration, i.e., on the effect of the relative FP lengths on perceived duration (Gamache et al., 2011; Grondin \& Rammsayer, 2003; Mo \& George, 1977). Some of these studies did identify factors influencing the FP effect that can be construed as modulators of prior temporal uncertainty. However, the factors that were identified only provide information given by external means and not by the distribution of FPs itself. Mo and George (1977) gave the participants information about the FP by associating each possible FP with tones of different pitches. Gamache et al. (2011) manipulated prior information about the FP in a similar manner by informing or not that the FPs were variable. The participants in the constant FP group who were falsely led to believe that the FP would be variable showed a consistent pattern of temporal underestimation compared with those that were given no prior information. What remains to be seen is whether knowledge of the distribution of FPs acquired through statistical learning can act as prior temporal information of the FP length and whether the uncertainty associated with this information is related to the variability of the FPs.

\section{The present study}

If the effect of FP length on time perception can be explained adequately by temporal preparation, the perceived duration of target time intervals is expected to vary as a function of the preceding FP. This should hold only for trials where FPs vary randomly from trial to trial. In order to test this assertion, both constant and variable FP designs were used in the present experiment. Furthermore, if temporal preparation is influenced by the prior temporal uncertainty about the FP length, the temporal underestimation of the target intervals following the shortest FP should be magnified by an increase of the within-block variability of the FP. As a result, the positive relationship between perceived duration and FP length should increase with within-block FP variability.

\section{Method}

\section{Participants}

Eighteen participants ( 7 females and 11 males, aged $24.6 \pm$ 5.1 years) were recruited through an email sent to all Laval University students and employees. All participants received $\$ 25$ (Canadian) for taking part in this experiment. All participants had normal hearing.

\section{Apparatus and stimuli}

The presentation of the auditory intervals and the recording of the participant's responses were computer controlled, using E- 
prime 2.0 software. The auditory stimuli were $1-\mathrm{kHz}$ square wave tones presented binaurally through headphones (Sony MDR-V600) with an intensity of approximately $70 \mathrm{~dB}$ SPL. The intervals to be discriminated were silent durations (empty intervals) marked by 10 -ms tones.

\section{Procedure}

Participants were seated at a table in a dimly lit, sound attenuated room, and were run individually. Their task was to decide whether the presented empty interval was shorter or longer than the 100-ms standard interval by pressing "1" (short) or " 3 " (long) on the computer keyboard. The instructions for participants emphasized accuracy, that is, there was no requirement to respond quickly.

An experimental session consisted of three sets of four experimental blocks with 96 trials per block. Each set of four blocks was initiated by 10 presentations of a $100-\mathrm{ms}$ standard interval. On each trial, an empty interval shorter or longer than the 100-ms standard interval was presented. There were four shorter durations $(79,85,91$, and $97 \mathrm{~ms})$ and four longer durations $(103,109,115$, and $121 \mathrm{~ms})$. Within each block, each of these eight intervals was presented 12 times in a random order. There were two sessions, one for the constant FP condition, and one for the variable FP condition. The sessions were completed on separate days and were counterbalanced across participants. Each experimental session lasted approximately 60 minutes.

Participants were tested in all conditions derived from interaction of the following three within-subjects variables: FP type (variable vs. constant), FP range (150, 300 or $900 \mathrm{~ms})$, and FP rank $(1,2,3,4) .{ }^{1}$ In the 150 -ms range condition, the FPs from rank 1 to 4 lasted 975, 1025, 1075, and $1125 \mathrm{ms,}$ respectively; it lasted 900, 1000, 1100, and $1200 \mathrm{~ms}$ in the 300 -ms range condition; and 600, 900, 1200, and $1500 \mathrm{~ms}$ in the 900-ms range condition. Also, the FPs were defined as the period of time from completion of the participant's response on the preceding trial to the onset of the first stimulus presentation on the following trial.

Each set of four blocks corresponded to one of three range conditions. Within a set of blocks, the four FPs from the respective range were presented. For instance, in the set of blocks associated with the 150-ms range condition, 975-, 1025-, 1075-, and 1125-ms FPs were used. In the constant FP condition, each block corresponded to one of the FP conditions. During the variable FP session, the FP could vary from trial to trial. The probability distribution of the FPs in the variable conditions was uniform, meaning that each of the four possible FP lengths in a block appeared exactly $25 \%$ of

${ }^{1}$ FP rank refers to the rank of a given FP within a set of blocks. For the variable FP condition, however, it is equivalent to the FP rank within a block. the time. The order of the different levels of range and the order of the FP conditions in the constant condition were partially counterbalanced across participants. Also, participants were given the opportunity to take a break between each set of blocks.

\section{Data analyses}

For each participant and for each FP, an 8-point psychometric function was traced, plotting the eight comparator intervals on the $x$-axis and the probability of responding "long" on the $y$ axis. The cumulative normal distribution was then fitted to the resulting curves.

Two indices of performance were estimated for each psychometric function, one for perceived duration and one for sensitivity. The first was the BP, which can be defined as the target duration corresponding to. 50 probability of "long" responses on the psychometric function. When the psychometric function takes the form of a cumulative Gaussian, the BP is simply the mean parameter. The observed shift of the BP for different FP lengths and conditions can be interpreted as an indication of differences in perceived duration such that longer perceived durations are reflected by smaller BP values (Fig. 1A and B). This interpretation assumes that participants employed the same response criterion for all conditions and that the differences in the distributions of "long" and "short" responses depend only on the perceived duration in each condition (Grondin, 1998, 2008).

Measuring temporal sensitivity (or level of duration discrimination), the other dependent variable in the present study, entailed estimating of the standard deviation of the psychometric function (Fig. 1C and D). By dividing this value by the actual duration of the standard (100 ms), we obtain the Weber ratio (WR). Using WR is a common procedure to index temporal sensitivity, with smaller WRs reflecting greater sensitivity (Grondin, 2008).

To assess the goodness-of-fit of the estimated psychometric functions, the $R^{2}$ statistic was computed. Four participants exhibited such poor fit indices (all mean $R^{2}<0.78$ ) that their data were excluded from further analyses. ${ }^{2}$

We conducted two 2 (FP type $) \times 3$ (FP range $) \times 4$ (FP rank $)$ factorial ANOVA, with a fully within-subject design: one taking BPs as a dependent variable data and the other taking WR. For both ANOVAs, we ran Mauchly's test of sphericity and we applied the Greenhouse-Geisser correction whenever it

${ }^{2}$ The very low R2 values of the fits showcase the fact that the performance of these participants was near chance level. In fact, the mean proportion of correct responses for these participants was $55 \%, 60 \%, 61 \%$ and $66 \%$. At such low levels of performance, indices obtained from psychometric function fitting are not reliable enough to be included in subsequent analyses. 
was needed. Finally, to test the hypothesis regarding the FP range modulation of the random FP effect, we conducted a polynomial contrast analysis. More specifically, we tested the statistical significance of the interaction contrasts comparing the linear trends of FP rank on BPs for each FP range condition. If the magnitude of the FP effect increase with withinblock FP variability, then the negative linear trend should be greater for the 900-ms FP range conditions than for the 300- or 150 -ms.

\section{Results}

Mean BPs as a function of the three independent variables are illustrated in Fig. 2. A rapid visual inspection of the figure suggests that the BP decreases as a function of the FP length in the variable FP condition, but not in the constant FP condition. Indeed, in the variable condition, the magnitude of the FP effect seems to be related to the range condition.

The ANOVA on BPs showed that only one main effect was significant, that of FP rank, $F(1.78,23.17)=10.24, p<0.001$, $\eta_{p}^{2}=0.44, \varepsilon_{\mathrm{gg}}=0.59$. Neither the FP range, $F(2,26)=2.36, p$ $=0.11, \eta_{p}^{2}=0.15$, nor the FP type effects, $F(1,13)=2.89, p=$ $0.11, \eta_{p}^{2}=0.18$, were statistically significant. As for the interaction effects, neither the three-way interaction effect, $F(1.90,24.74)=2.56, p=0.10, \eta_{p}^{2}=0.16, \varepsilon_{\mathrm{gg}}=0.32$, nor the FP type $\times$ FP range effect, $F(1.30,16.92)=2.56, p=0.12$, $\eta_{p}^{2}=0.16, \varepsilon_{\mathrm{gg}}=0.65$ were statistically significant. However, the FP range $\times$ FP rank effect, $F(6,78)=5.12, p<0.001, \eta_{p}^{2}=$ 0.28 , as well as the FP type $\times \mathrm{FP}$ rank effect, $F(1.64,21.29)=$ $17.17, p<0.001, \eta_{p}^{2}=0.57, \varepsilon_{\mathrm{gg}}=0.65$, were significant.

Because the FP type was involved in one of the significant interaction effects, the range and rank effects were evaluated separately for the constant and for the variable FP conditions. An adjusted alpha value of 0.025 was selected in order to control for type I error. For each FP type, a 3 (FP range) $\times 4$ (FP rank) factorial ANOVA, with a fully within-subject design, was conducted on the mean BPs.

The left panel of Fig. 2 shows the BP for each constant FP condition. The ANOVA revealed a significant $\mathrm{FP}$ range effect, $F(2,26)=4.55, p=0.02, \eta_{p}^{2}=0.26$, but no FP rank effect, $F(1.82,23.64)=1.23, p=0.31, \eta_{p}^{2}=0.087, \varepsilon_{\mathrm{gg}}=0.61$ and no interaction, $F(2.51,32.58)=0.18, p=0.88, \eta_{p}^{2}=0.014, \varepsilon_{\mathrm{gg}}=$ 0.42 .

The BPs of the variable FP conditions are shown on the right panel of Fig. 2. The ANOVA related to the variable FP condition revealed that the main effect of FP rank was significant, $F(1.48,19.20)=22.16, p<0.001, \eta_{p}^{2}=0.63, \varepsilon_{\mathrm{gg}}=$ 0.49 , and that the main effect of FP range was not, $F(1.24$, $16.06)=1.13, p=.32, \eta_{p}^{2}=0.08, \varepsilon_{\mathrm{gg}}=0.62$. The interaction effect also was significant, $F(2.32,30.16)=8.51, p<0.001$, $\eta_{p}^{2}=0.40, \varepsilon_{\mathrm{gg}}=0.39$.

Overall, there is a negative relationship between relative rank in a FP set and BPs and, thus, a positive relationship between relative rank and perceived duration. When we decomposed the FP rank $\times$ FP range interaction by testing the simple effect of FP rank at each FP range, we found that they were all statistically significant $(F(1.32,17.21)=5.49, p$ $=0.024, \eta_{p}^{2}=0.30, \varepsilon_{\mathrm{gg}}=0.44$, for the $150-\mathrm{ms}$ range condition; $F(3,39)=13.48, p<0.001, \eta_{p}^{2}=0.51$ for the $300-\mathrm{ms}$ condition; $F(1.21,15.74)=19.33, p<0.001, \eta_{p}^{2}=0.60, \varepsilon_{\mathrm{gg}}=$ 0.40 . for the $900-\mathrm{ms}$ range). The polynomial contrast analysis reveals that, for all three FP range conditions, the linear trend was significant and negative $(C=-5.56, t(39)=-4.02, p<$ $0.001, d=1.52, r_{a l}^{2}=0.98$ for the 150-ms range; $C=-5.30$, $t(39)=-5.49, p<0.001, d=2.07, r_{a l}^{2}=0.74$ for the $300-\mathrm{ms}$

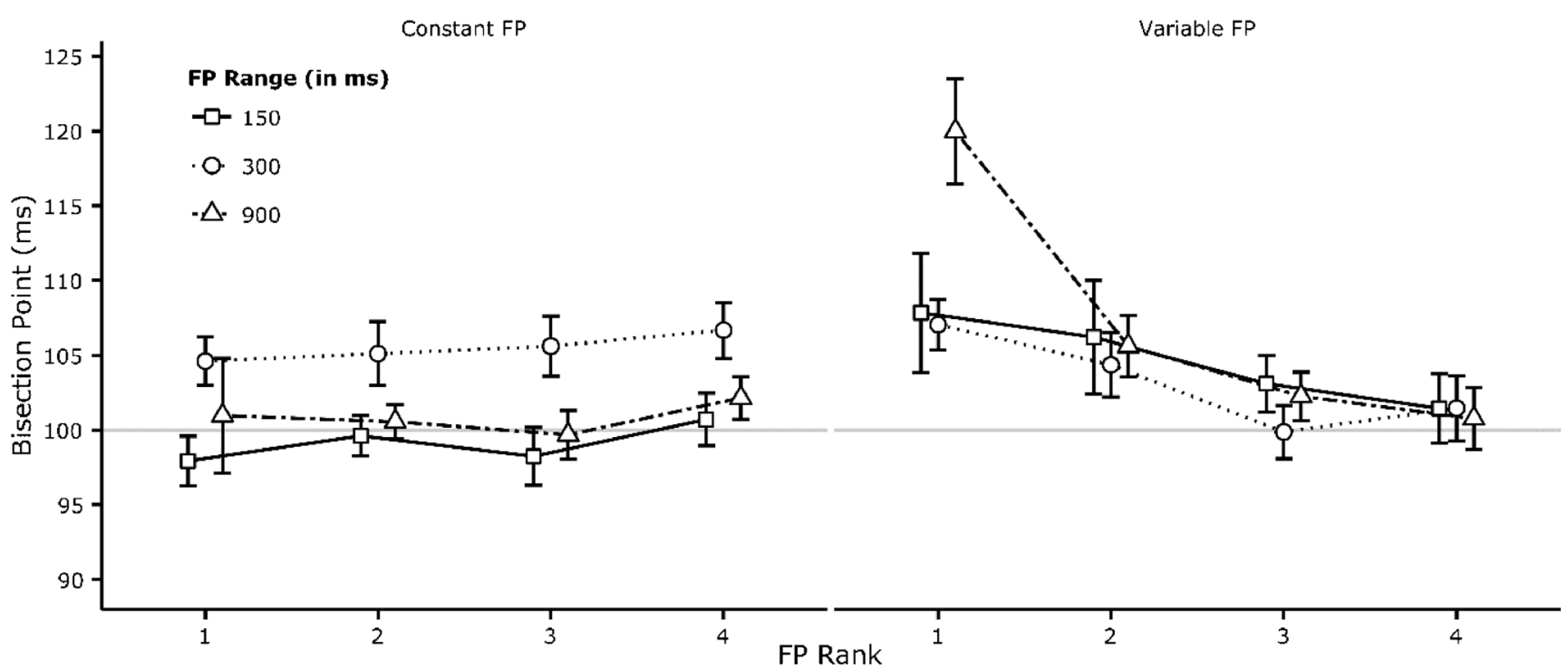

Fig. 2 Mean bisection point (ms) in the constant (left panel) and variable (right panel) FP conditions as a function of the FP range and the FP rank ( \pm standard error of the mean) 
range and $C=-15.26, t(39)=-6.83, p<0.001, d=2.58, r^{2}$ al $=0.80$ for the $900-\mathrm{ms}$ condition). Moreover, the linear trend varied as a function of FP range. Indeed, there was a statistically significant difference between the linear contrast at $150 \mathrm{~ms}$ and the one at $900 \mathrm{~ms}, C=4.85, t(143)=2.96, p=$ $0.0036, d=1.12, r_{a l}^{2}=0.12$, as well as between the one at 300 and at $900 \mathrm{~ms}, C=4.98, t(143)=3.04, p=0.0029, d=1.15$, $r_{a l}^{2}=0.12$. The difference between the linear trends at 150 and $300 \mathrm{~ms}$, however, was not statistically significant, $C=-0.13$, $t(143)=-0.079, p=0.94, d=0.03, r_{a l}^{2}<0.001$.

One should note that the relationship between FP rank and BP is not strictly linear, but rather quadratic or exponential. We tested the second and third-order polynomial contrasts for each range condition. We found no statistically significant cubic trend at any of the FP range conditions ( $p \mathrm{~s}>0.025)$. There were, however, statistically significant quadratic contrasts in the 300-ms FP range condition, $C=2.13, t(39)=2.47$, $p=0.018, d=0.93, r_{a l}^{2}=0.15$, as well as in the $900-\mathrm{ms}$ condition, $C=6.44, t(39)=3.22, p=0.0026, d=1.22, r^{2}{ }_{a l}$ $=0.18$.

Another feature of the data is that, whereas all FP ranges seem to produce equivalent BPs at the second-, third-, and fourth-rank conditions, the 900-ms FP range seems to produce significantly higher BPs at the first-rank condition than at the other two FP ranges. We decomposed this interaction effect by looking at the range effect for each rank (1 to 4). For Rank 1 (the shortest FPs), the range effect was significant, $F(2,26)=$ $6.17, p=0.006, \eta_{p}^{2}=0.32$. For Ranks 2, 3, and 4, no effect was significant ( $p$ values $=0.74,0.27$, and 0.95 , respectively).

As for the results related to the sensitivity levels, we analyzed the WR data using the same 2 (FP type) $\times 3$ (FP range) $\times$ 4 (FP rank) factorial ANOVA, with a fully within-subject design. The relevant data are illustrated in Fig. 3. It can be seen on that graph that the only difference appears to be in the

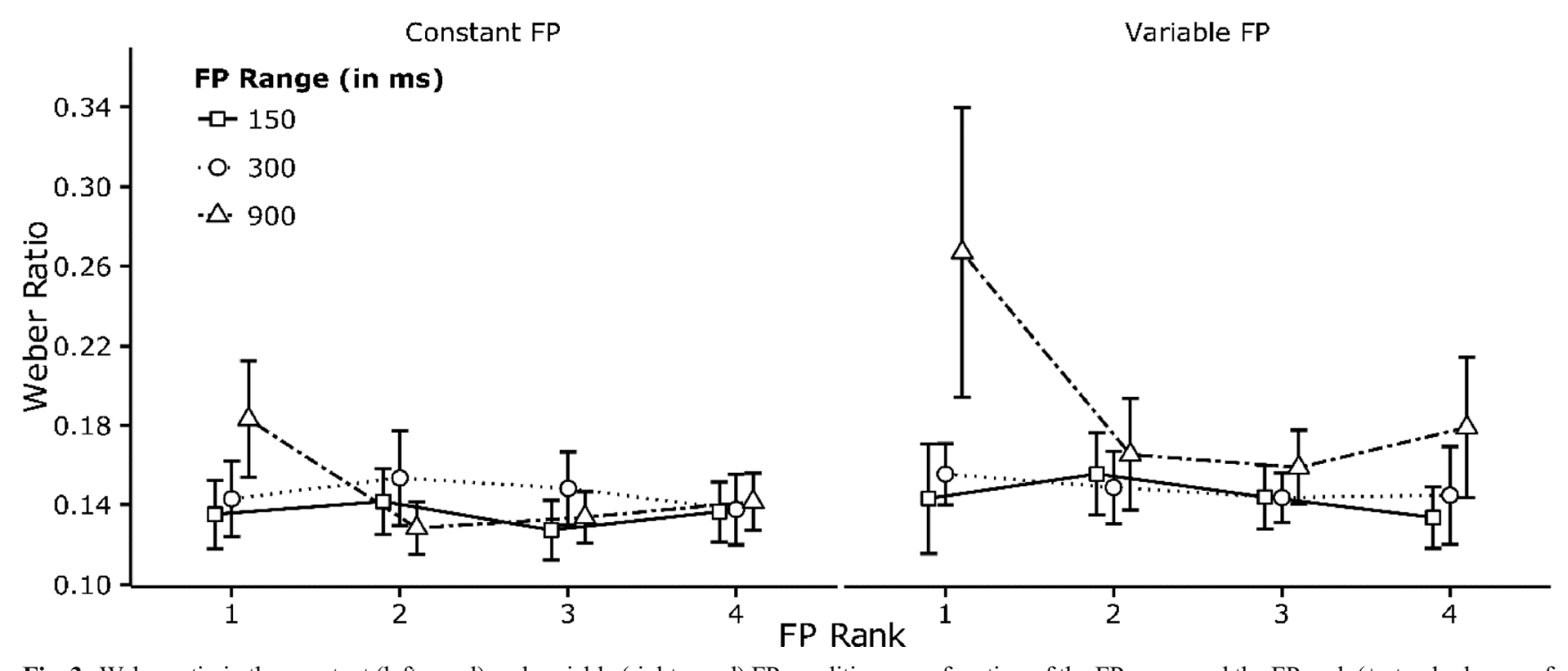

Fig. 3 Weber ratio in the constant (left panel) and variable (right panel) FP conditions as a function of the FP range and the FP rank ( \pm standard error of the mean) shortest variable FPs. Indeed, the main effect of rank on WR was significant, $F(3,39)=4.41, p<0.001, \eta_{p}^{2}=0.25$, as well as the FP rank $\times$ FP range interaction, $F(2.72,35.37)=3.75, p$ $=0.022, \eta_{p}^{2}=0.22, \varepsilon_{\mathrm{gg}}=0.45$. For all other effects, $p>0.05$. The analysis of the simple effects for each FP rank reveals that the simple main effect of FP range at the lowest FP rank was marginally significant (at the $0.05 / 4$ level), $F(1.12,14.51)=$ $5.08, p=0.037, \eta_{p}^{2}=0.28, \varepsilon_{\mathrm{gg}}=0.56$. Concerning the simple effects across FP ranges, the only statistically significant effect was that of FP rank and occurred at the 900-ms range condition, $F(1.24,16.07)=6.69, p=0.015, \eta_{p}^{2}=0.34, \varepsilon_{\mathrm{gg}}=0.41$.

\section{Discussion}

The main objective of the current study was to submit the temporal preparation account of the variable FP effect on perceived duration to further empirical testing. If this explanation is valid, then the following two-part prediction should have been confirmed by the results of the present study: 1) in variable FP conditions, the magnitude of the relative FP length effect on perceived duration of a target interval should be related positively with the within-block variability (or range, in the case of uniformly distributed FP) of FPs, and 2) in constant FP conditions, there should be no relative FP length effect since the within-block variability of FPs is 0 . Both parts of this hypothesis were at least partially confirmed by the results of the present experiment. Indeed, in the variable FP conditions, BPs decreased monotonically as relative FP length (measured as FP rank) increased. This is consistent with the results from the previous studies on the subject of FP effects on time perception (Gamache et al., 2011; Grondin \& Rammsayer, 2003; Mo \& George, 1977) and on RT (Niemi \& Näätänen, 1981; Requin, Granjon, Durup, \& Reynard, 
1973). Also, the magnitude of that decrease was significantly larger for the 900-ms FP range conditions than for the 150-ms and 300-ms ones. Moreover, the largest temporal underestimation occurred with the shortest level of the most variable FP condition. This is largely consistent with the findings in the literature regarding the modulation of the variable FP effect on RT by FP variability (Elliott, 1973) as well as the general temporal preparation account of the FP effect on RT (Los \& Van den Heuvel, 2001; Los \& Horoufchin, 2011). Finally, as the absence of statistically significant effects related to FP rank attests to, perceived duration was largely unaffected by the relative FP length in the constant FP conditions.

Together, these findings thus bring further credibility to the temporal preparation explanation of the FP effect on time perception (Gamache et al., 2011; Grondin \& Rammsayer, 2003; Mo, 1990; Mo \& George, 1977). When target time intervals follow randomized FPs, it creates uncertainty regarding the time-of-arrival of the onset of the target interval. The greater that uncertainty is, the less prepared the observer is to start processing the target interval. In terms of the SET (Gibbon et al., 1984), it means that greater temporal uncertainty about the FP length delays the closing of the attentional switch and, thus, decreases the amount of pulses ultimately collected by the accumulator.

Temporal uncertainty is mitigated by both prior and posterior information regarding the length of the current FP (Mo, 1990). As explained previously, prior temporal information refers to the knowledge one extracts about the FPs before the start of the trial. Posterior information is defined as the information one can deduce (consciously or not) about the current FP and is generally thought to be the inferred subjective probability that the target stimulus will appear shortly. By increasing the variability of the random FPs, prior information about the FP is made less effective at mitigating temporal uncertainty about the FP and thus at predicting the onset of the target interval. That would explain why the largest BP and, thus, the shortest perceived duration, were produced at the shortest relative FP length (rank 1) and largest FP range (900 ms). Indeed, it is at that point that both posterior and prior information are at their lowest level. However, as relative FP length increases, prior information becomes less and less important for predicting the onset of the target interval such that, for the longest relative FP length, performance for all FP ranges reach the same level: veridical duration (BP of $100 \mathrm{~ms}$ ).

In the context of the preparation explanation of the FP effect, the presence of a significant main effect of FP range on perceived duration in the constant conditions remains puzzling since the within-block range of the FP was always 0 . However, one must note that in the current design, the FP range factor is defined as the range of FPs presented within a set of blocks and not within each individual block. This means that, in the case of constant FP conditions, there could be an influence of the within-set context on the perceived duration of target intervals. It remains surprising, however, that it is the middle FP range $(300 \mathrm{~ms})$ that led to the shortest perceived duration and that the other FP ranges led to essentially veridical perceived duration. Indeed, while it is not surprising that prior information about the FP duration could have an effect on temporal uncertainty and, ultimately, on perceived duration, in constant FP conditions, one would expect that the largest FP ranges would induce the largest temporal underestimation amongst the target intervals.

The analysis of sensitivity — of the Weber Ratio (WR) - in the variable FP conditions show a pattern qualitatively similar to that of the BPs: the WR was maximal (and sensitivity, minimal) at the shortest of the most variable FP condition (rank 1; range $900 \mathrm{~ms}$ ) and was roughly equal for all other variable FP conditions. The same pattern is found in the constant FP conditions, albeit with a smaller maximal value of WR. It would thus seem that the absolute value of the FP length - and not the relative length — influences the precision of the participants' temporal estimates regardless of FP type. This kind of dissociation between the FP effect on perceived duration and that on sensitivity also was observed in Grondin and Rammsayer (2003) and Gamache et al. (2011). It was hypothesized that FP length influences perceived duration on a more basic (i.e., perceptual) level while they influence sensitivity levels by interfering with higher-order component of the discrimination processes (i.e., decisional and memory components). This explanation seems to hold for the present data but would certainly warrant further testing and elaboration in the future.

\section{Conclusions}

The results of the present study lend further credence to the temporal preparation account of the FP effect on time perception. They provide evidence for the existence of a previously unknown correspondence between the effect of variable FP length on RT and that on perceived duration: greater FP variability within each block results in greater variable FP effect. They also confirm that relative FP length has no influence on perceived duration. However, it seems that the range of FP presented within a set of blocks can have an influence on perceived duration, even though that influence is not straightforward. In brief, it was shown that prior information about the FP distribution can modulate the FP effect on time perception.

Authors Note This research was made possible by a research grant awarded to SG by the Natural Sciences and Engineering Council of Canada. The authors thank Sander Los, James Broadway, and one anonymous reviewer for their numerous comments and very helpful suggestions on an earlier draft of this paper. This study was presented at the $55^{\text {th }}$ Annual Meeting of the Psychonomic Society, Los Angeles, CA, November 2014. 


\section{References}

Bausenhart, K. M., Rolke, B., \& Ulrich, R. (2008). Temporal preparation improves temporal resolution: Evidence from constant foreperiods. Perception and Psychophysics, 70, 1504-1514.

Bendixen, A., Grimm, S., \& Schröger, E. (2005). Human auditory eventrelated potentials predict duration judgments. Neuroscience Letters, 383, 284-288.

Block, R. A., \& Zakay, D. (1996). Models of psychological time revisited. In H. Helfrich (Ed.), Time and Mind (pp. 171-195). Kirkland, WA: Hogrefe und Huber.

Block, R. A., \& Zakay, D. (1997). Prospective and retrospective duration judgments: A meta-analytic review. Psychonomic Bulletin \& Review, 4, 184-197. doi:10.3758/BF03209393

Brown, S. W. (1997). Attentional resources in Timing: Interference effects in concurrent temporal and nontemporal working memory tasks. Perception \& Psychophysics, 59, 1118-1140.

Casini, L., Macar, F., \& Grondin, S. (1992). Time estimation and attentional sharing. In F. Macar, V. Pouthas, \& W. Friedman (Eds.), Time, Action, Cognition: Towards Bridging the Gap (pp. 177-180). Dordrecht, Netherlands: Kluwer.

Drazin, D. H. (1961). Effects of foreperiod, foreperiod variability, and probability of stimulus occurrence on simple reaction time. Journal of Experimental Psychology, 62, 43-50. doi:10.1037/ h0046860

Elliott, R. (1973). Some confounding factors in the study preparatory set in reaction time. Memory \& Cognition, 1, 13-18. doi:10.3758/ BF03198063

Gamache, P., Grondin, S., \& Zakay, D. (2011). The impact of attention on the internal clock in prospective timing: Is it direct or indirect? In A. Vatakis, A. Esposito, M. Giagkou, F. Cummins, \& G. Papadelis (Eds.), Time and Time Perception 2010, LNAI 6789 (pp. 137150). Berlin: Springer-Verlag.

Gibbon, J., Church, R. M., \& Meck, W. H. (1984). Scalar timing in memory. In J. Gibbon \& L. Allan (Eds.), Timing and time perception (Annals of the New York Academy of Sciences (Vol. 423, pp. 52-77). New York: New York Academy of Sciences.

Grondin, S. (1998). Judgments of the duration of visually marked empty time intervals: Linking perceived duration and sensitivity. Perception \& Psychophysics, 60, 319-330. doi:10.3758/ BF03206040

Grondin, S. (2008). Methods for studying psychological time. In S. Grondin (Ed.), Psychology of Time (pp. 51-74). Bingley, UK: Emerald Group Publishing.

Grondin, S., \& Macar, F. (1992). Dividing attention between temporal and nontemporal tasks: A performance operating characteristic-
POC - analysis. In F. Macar, V. Pouthas, \& W. Friedman (Eds.), Time, action, cognition: Towards bridging the gap (pp. 119-128). Dordrecht, Netherlands: Kluwer.

Grondin, S., \& Rammsayer, T. (2003). Variable foreperiods and temporal discrimination. The Quarterly Journal of Experimental Psychology Section A: Human Experimental Psychology, 56, 731-765. doi:10. 1080/02724980244000611

Karlin, L. (1959). Reaction time as a function of foreperiod duration and variability. Journal of Experimental Psychology, 58, 185-191. doi: 10.1037/h0049152

Klemmer, E. T. (1956). Time uncertainty in simple reaction time. Journal of Experimental Psychology, 51, 179-184. doi:10.1037/h0042317

Los, S. A., \& Horoufchin, H. (2011). Dissociative patterns of foreperiod effects in temporal discrimination and reaction time tasks. Quarterly Journal of Experimental Psychology, 64, 1009-1020. doi:10.1080/ 17470218.2010 .532225

Los, S. A., \& Van Den Heuvel, C. E. (2001). Intentional and unintentional contributions of nonspecific preparation during reaction time foreperiods. Journal of Experimental Psychology: Human Perception and Performance, 27, 370-386. doi:10.1037//00961523.27.2.370

Macar, F., Grondin, S., \& Casini, L. (1994). Controlled attention sharing influences time estimation. Memory \& Cognition, 22, 673-686.

Mo, S. S. (1990). Time reversal in human cognition: Search for a temporal theory of insanity. In R. A. Block (Ed.), Cognitive Models of Psychological Time (pp. 241-254). Hillsdale NJ: Lawrence Erlbaum Associates.

Mo, S. S., \& George, E. J. (1977). Foreperiod effect on time estimation and simple reaction time. Acta Psychologica, 41, 47-59. doi:10. 1016/0001-6918(77)90010-5

Niemi, P. (1979). Stimulus intensity effects on auditory and visual reaction processes. Acta Psychologica, 43, 299-312. doi:10.1016/00016918(79)90038-6

Niemi, P., \& Näätänen, R. (1981). Foreperiod and simple reaction time. Psychological Bulletin, 89, 133-162. doi:10.1037//0033-2909.89.1. 133

Requin, J., Granjon, M., Durup, H., \& Reynard, G. (1973). Effects of a timing signal on simple reaction time with a rectangular distribution of foreperiods. Quarterly Journal of Experimental Psychology, 25, 344-353. doi:10.1080/14640747308400355

Zakay, D., \& Block, R. A. (1996). The role of attention in time estimation processes. In M. A. Pastor \& J. Artieda (Eds.), Time, Internal Clocks and Movement (pp. 143-164). Amsterdam: Elvesier.

Zakay, D., \& Block, R. A. (1997). Temporal Cognition. Current Directions in Psychological Science, 6, 12-16. doi:10.1111/14678721.ep11512604 